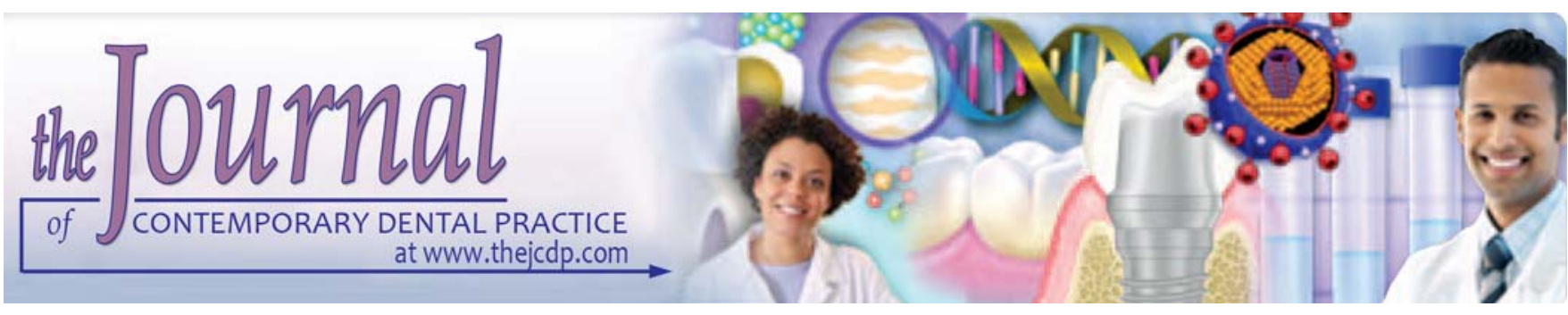

\title{
Effects of Commonly Consumed Beverages on Surface Roughness and Color Stability of the Nano, Microhybrid and Hybrid Composite Resins: An in vitro Study
}

P Sarveshwar Reddy, KL Sunil Tejaswi, Suneeth Shetty, BM Annapoorna, Sudarshan C Pujari, HM Thippeswamy

\section{ABSTRACT}

Background: Consumption of certain beverages may affect the esthetic and physical properties of the resin composite, thereby undermining the quality of restorations.

Aim: To analyze the effect of three beverages (cola, coffee, tea) on color stability and surface roughness of three different types of resin composites at various time intervals in vitro.

Materials and methods: Nano, microhybrid and hybrid resin composites were used. Each material was randomly divided into four equal subgroups of 10 samples each according to the beverages used (cola, coffee, tea, distilled water). The samples were immersed in each beverage for 1,15 and 30 days. Surface roughness and color changes measurements were noted at the baseline-the first, fifteenth and thirteenth day.

Results: It was found that nanoresin composite followed by microhybrid and hybrid showed least surface roughness and color change. The Coke beverage subgroup showed more surface roughness and the subgroup coffee has shown more color changes with respect to other subgroups.

Conclusion: All specimens showed discoloration after completion of the test period which was visually perceptible and clinically unacceptable.

At the end of 30th day, among the materials, nanofilled composite resin showed comparatively less surface roughness and color change than microhybrid and hybrid composite resins.

Keywords: Profilometer, Spectrophotometer, Nanofilled composite resin, Microhybid composite resins, Hybrid composite resin, Coffee.

How to cite this article: Reddy PS, Tejaswi KLS, Shetty S, Annapoorna BM, Pujari SC, Thippeswamy HM. Effects of Commonly Consumed Beverages on Surface Roughness and Color Stability of the Nano, Microhybrid and Hybrid Composite Resins: An in vitro Study. J Contemp Dent Pract 2013;14(4): 718-723.

Source of support: Nil

Conflict of interest: None declared

\section{INTRODUCTION}

Esthetics has played a major role in the development of dentistry and dental research. The trend toward a natural look has paved the way for the development of tooth colored restoratives that simulate the tooth as closely as possible. ${ }^{1,2}$

Increasing demand for esthetic dentistry has fueled a rapid development of new restorative resins. ${ }^{3}$ The newer universal composite systems combine the properties of the earlier hybrid and microfilled composites. ${ }^{4}$ Modifications of fillers in conventional resin-based composites have improved their mechanical properties and esthetic performances. One of the most significant modifications in recent years has been the application of nanotechnology to resin composites. ${ }^{5}$

Resin nanocomposites (NCs) have many advantages such as increased mechanical properties, improved optical properties, better gloss retention and diminished wear. ${ }^{5}$ Thus, they provide an ideal material for direct esthetic anterior restorations. ${ }^{4}$

Discoloration of tooth colored resin-based materials may be caused by either intrinsic or extrinsic factors.

The intrinsic factors depend on the composition of the matrix (percentage of Bis-GMA, UDMA), the type of bonding between the fillers and matrix, etc.

Extrinsic factors such as adsorption or absorption of extrinsic stains pose a major problem for esthetic restorations. Erikson and Nordbo et al suggested that retention of colored substances from dietary constituents significantly contribute to the formation of extrinsic stains. Certain unique topographic patterns are seen with respect to beverage consumption in India, with coffee being consumed more frequently in the South India, while tea is consumed more frequently in North India. Also, there is 
higher consumption of alcoholic and erated beverages such as Coke among the younger generation. These beverages are commonly consumed frequently and in-between meals, thereby predisposing composite restorations for discoloration. $^{7-9}$

Surface roughness is one of the reasons for exterior discoloration. ${ }^{10}$ The water absorption ability of resin composites may also be influenced by their chemical composition, which represents an important feature of the color stability and surface integrity of composites. ${ }^{11}$ During wear of dental resin composites, inorganic fillers debond from the resin matrix and leave a void, increasing the surface roughness and forming a surface susceptible to exterior stain. $^{12}$

\section{AIM}

The purpose of this study is to evaluate the surface roughness and color stability of nanofilled, microhybrid composite and hybrid composite restorative materials after exposing them to the three commonly consumed beverages- tea, coffee and Coke, using a profilometric and spectrophotometric analysis.

\section{MATERIALS AND METHODS}

Three resin composites used in this study were:

Group 1: Nanoresin composite (Filtekz 350, A2 SHADE, 3MESPE St. Paul, MN, USA).

Group 2: Microhybrid resin composite (Filtek Z250, A2 SHADE, 3MESPE).

Group 3: Hybrid resin composite (Filtek Z100, A2 SHADE, 3MESPE).

The beverages used in the study were Coca Cola (Hindustan Coca-Cola Beverages Pvt Ltd, India), coffee (Nestle India, India) and tea (Tata Tea, Tata Global Beverages Ltd, India).

Distilled water was used as the control.

\section{Method of Sample Preparation}

The test samples in this study were in the form of disks measuring $10 \mathrm{~mm}$ in diameter and $2 \mathrm{~mm}$ in thickness. To standardize disk size, they were prepared using a Teflon mold of this size.The mold was filled with resin composite, covered with clear celluloid strips at the bottom and top of the hole. To achieve uniform disk specimen thickness it was pressed between two glass slides. Each specimen was cured for 40 seconds with LED curing light (Dentsply, USA). After curing, the specimen was removed and polished with composite finishing and polishing kit (Shofu, Japan).
For each resin composite to be tested, 40 disk specimens were made. Therefore, the total sample size is was 120 .

1. Group 1: 1A (distilled water), 1B (coffee), 1C (tea), 1D (cola).

2. Group 2: 2A (distilled water), 2B (coffee), 2C (tea), 2D (cola).

3. Group 3: 3A (distilled water), 3B (coffee), 3C (tea), 3D (cola).

\section{Method of Test Solution Preparation}

In order to standardize the preparation of tea and coffee solutions, an electronically controlled tea/coffee maker with time and temperature control was used. Distilled water was used to prepare tea and coffee to avoid any contamination from the water source.

The samples were stored in test tubes during the test period.

$\begin{array}{ll}\text { Volume of single test tube } & : 3 \mathrm{ml} \\ \text { Total no. of test tubes for tea } & : 15 \\ \text { Total volume of test tubes for tea } & : 15 \times 3=45 \mathrm{ml}\end{array}$

In order to avoid less volume of prepared solution during boiling due to vapor loss, additional 5 to $6 \mathrm{ml}$ distilled water is added. The tea powder was measured with a spoon having of $25 \mathrm{gm}$. The tea solution was then prepared with the help of electronic tea/coffee maker. The same procedure mentioned above was followed for the preparation of the coffee solution. For the other two test solutions, i.e. Coke and distilled water, same measurements were carried out and the test solutions were stored in test tubes.

The samples were stored for the study period of 30 days. The color change was measured at 24 hours, 15 and 30 days after immersion. The test solutions were changed every seventh day following the same procedure as mentioned previously. Surface roughness of the materials was tested using a profilometer.

At the time of color measurement, the samples were removed from the test solutions, dipped in distilled water 10 times, wiped with tissue paper and placed on a thermacol platform with a slot in which the study samples were place. The platform also had a slot for the spectrophotometer used for the color measurement. The spectrophotometer was calibrated by measuring against standard white background provided by manufacturer, a process known as 'Zeroing'. The readings were taken under the illumination of D50, which is equal to natural daylight.

The spectrophotometer software calculates the color measurement $(\Delta \mathrm{E})$ using the following formula:

$$
(\Delta \mathrm{E})=\left(\Delta \mathrm{L}^{2}+\Delta \mathrm{b}^{2}+\Delta \mathrm{a}^{2}\right)^{1 / 2}
$$


L - lightness of color and ranges between 0 (dark) and 100 (White)

a - color on red-green axis

b - blue part of the color

Statistical methods applied: Mean and standard deviation was used for descriptive statistics. Paired t-test was used to calculate the mean surface roughness and color difference between baseline 24 hours, 15 days and 1 month. SPSS V 17 was used for statistical analysis. A p-value $<0.05$ was considered for statistical significance.

\section{RESULTS}

When changes in the surface roughness of the three composites was considered, the overall maximum surface roughness change took place in hybrid resin composites followed by microhybrid and nanocomposite resins.

The Coke beverage subgroup has shown more surface roughness compared to other beverage subgroup in different durations (24 hours, 15 days and 1 month) in all the composite resins. This is followed by coffee, tea and distilled water subgroup (Tables 1 and 3).

With respect to color change hybrid has shown more color changes in coffee beverages compared to other composites and other beverages. This is followed by nanoand microfilled composites in coffee beverages subgroups. Least color change took in distilled water (Tables 2 and 4).

Both surface roughness and color changes becoming more as the duration increases in all the beverages and in all composites.

\section{DISCUSSION}

Increasing demand for esthetics in dentistry has led to the rapid rate of development of new restorative resins. The newer universal composite systems combine the properties of earlier hybrid and microhybrid composites. ${ }^{4}$ However, success of any esthetic restoration depends, first on the color match and then on color stability of the material. ${ }^{3}$

Esthetic failure is one of the most common reasons for the replacement of restorations. A good combination of the tooth color and the initial color of the material before curing is an important clinical factor for a successful outcome. Nevertheless, this combination must remain after the material is completely cured and throughout life of the restoration. ${ }^{6}$

Geurtsen et al stated that the higher organic matrix of hybrid materials may be the reason of higher susceptibility to water absorption and material disintegration.

On the surface roughness of resin composites, is related to the composition and porosity of the material. ${ }^{13}$
Microhybrid composite materials incorporate a high volume fraction of filler particles with a narrow particle size distribution, and that the particles had a mean size below $1 \mu \mathrm{m} 20) .{ }^{14}$ Besides Bis-GMA, the matrix of this composite resin contains UDMA and modified urethane (Bis-EMA) to reduce the polymerization shrinkage and intrinsic stresses of the material. Furthermore, to enable a higher volume of fillers to be incorporated in the polymeric matrix, the microhybrid composite has $60 \%$ of small silica particles. ${ }^{15}$

Surface roughness of composites can reduce some mechanical properties such as hardness and increase the wear of restorations. Cola drinks have been found to cause the surface degradation of resin matrix and surface erosion of filler contents. In addition, the surface degradation of resin materials is related to the content of the fillers, distribution of the fillers, composition of the matrix resin, and the effect of silane surface treatment on the fillers. ${ }^{16}$ The increasing demand for esthetic dentistry has been coupled with a rapid development of new restorative materials. Nanohybrid ${ }^{17}$ composite is one of the newly developed esthetic restorative material, and it is currently being used for clinical work in operative dentistry. However, in the complex environment of the oral cavity (with exposure to alcohol, acids, and mechanical abrasion and temperature changes), nanohybrid composite are expected to undergo considerable degradation. ${ }^{18}$

Increase in surface roughness values after immersion in beverages, which could be attributed to water which can infiltrate and decrease the mechanical properties of the polymer matrix, by swelling and reducing the frictional forces between the polymer chains. ${ }^{19}$ Composite resins generally contain more organic matrix and thus may be more susceptible to water absorption and subsequent surface disintegration in an aqueous environment. ${ }^{20}$

The surface properties of a composite resin material, especially microhardness and roughness may be greatly affected by the general chemical composition of the beverages, the type of acid present in their formulation, and also the potency of the individual acidic ingredients. ${ }^{21}$

The staining observed in this study are those that occur on the surface. This is because of the hydrophobic monomers in composite resins. For instance, UDMA based monomers display lower staining values compared to other Dimethacrylate based monomer types. This may be accounted for low viscosity and low water absorption of UDMA and its successful polymerization. ${ }^{22}$ It was found that the incorporation of greater amount of TEGDMA resulted in an increase in water uptake in Bis-GMA based resins. ${ }^{6}$

According to the manufacturer's description, Filtek Z 350 is a nanofilled composite with filler content $79 \%$ w/w 

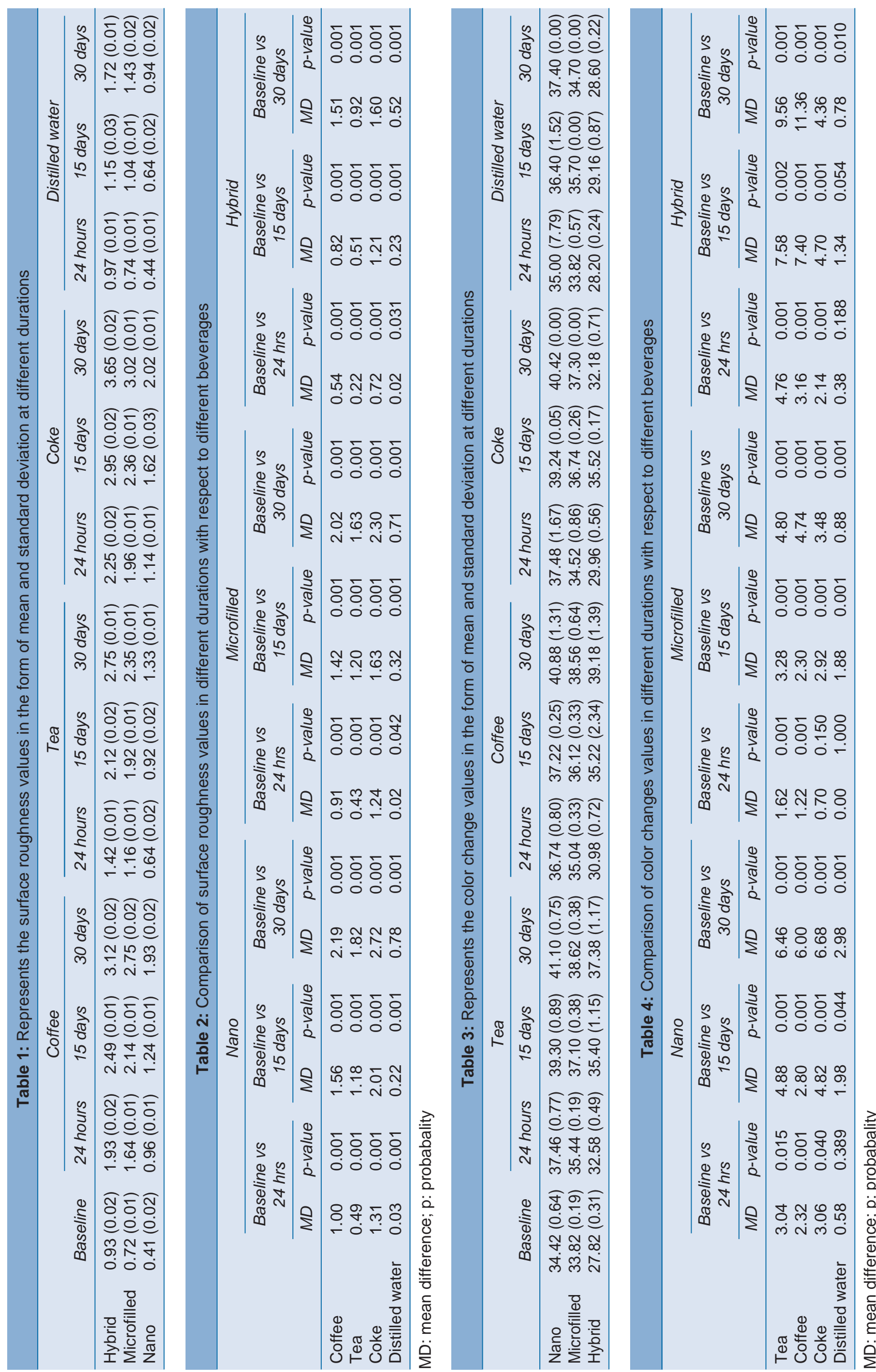
or $60 \% \mathrm{v} / \mathrm{v}^{5}$ Also monomers like Bis-GMA, Bis-EMA, UDMA and a small portion of a hydrophilic monomer TEGDMA are also present in its composition. The color change may be easily related to the nature of this resin matrix. The presence of low TEGDMA content may limit water uptake and consequently, color variation induced by the absorption of the staining solution. ${ }^{9}$

Likewise, the filler content seems to play an important role in color stability of the resin. ${ }^{8}$ As purported by the manufacturer, Filtek Z350 is a nanofilled composite resin with a primary $20 \mathrm{~nm}$ silica filler and loosely bonded cluster of Zirconia / Silica particles size ranging from 0.6 to $1.4 \mu \mathrm{m}$. In the present study, in order to simulate clinical situations, Sof-Lex contouring and polishing disks were used. Filtek Z350 has smaller filler particle size and hence, this material may have less surface roughness than other tested materials. ${ }^{6}$

It has been suggested that silanization of filler particles plays an important role in discoloration as does the type of resin used in resin based composites. This is due to a fact that silane has high water absorption levels. High staining values of Z250 and Z100 may be attributed to the high proportion of silane present in the structure of the material. ${ }^{23}$

The staining agents selected for the study are in common daily use and have a strong potential to stain tooth colored restorative materials. ${ }^{24}$ Generally, all staining agents stained all materials; coffee produced the most severe stain and cola the least. Since the $\mathrm{pH}$ of the staining agents in the present study ranges from 2.7 for cola to 5.38 for tea, a possibility is that the acidic $\mathrm{pH}$ may have had an effect on the structure of the materials. Although, cola had the lowest $\mathrm{pH}$ and it might damage the surface integrity of the materials, it did not produce as much discoloration as coffee and tea, which may be because of lack of a yellow colorant in cola. ${ }^{25} \mathrm{Um}$ and Ruyter et al (1991) showed that tea and coffee contain yellow colorants, which have different polarities. Higher polarity components (those in Tea) are eluted first and lower polarity components (those in Coffee) are eluted later. Therefore, discoloration by tea was due to adsorption of polar colorants onto the surface of materials which can be removed with daily cleaning habits as tooth brushing, etc. Whereas, discoloration by coffee was due both to adsorption and absorption of colorants. This absorption and penetration of colorants into the organic phase of the materials were probably due to compatibility of the polymer phase with the yellow colorants of coffee. ${ }^{8}$

\section{CONCLUSION}

Within the limits of present study, it can be concluded that:

1. All specimens showed discoloration after completion of the test period which was visually perceptible and clinically unacceptable.
2. Comparing within the materials, the hybrid composites showed more surface roughness than the microhybrid and nanofilled composites. This can be graded as , Hybrid $>$ Microhybrid $>$ Nanofilled.

3. Comparing within the test solutions, Coke has shown more surface changes than coffee and tea and coffee has shown more tendency for causing color change than tea and Coke. This can be graded as, Coffee $>$ Tea $>$ Coke $>$ Water.

4. At the end of 30th day, among the materials, nanofilled composite resin showed comparatively less surface roughness and color change than microhybrid and hybrid composite resins.

Hence, it should be noted that the patients should be made aware about the staining characteristics of the dilatory constituents particularly of beverages on tooth colored restorations. Also, clinicians should take every possible measure in order to minimize other contributing factors such as complete polymerization, incomplete finishing and polishing of the composite resins. All these, in turn, will help in prolonging the color retainability of the tooth colored restorative materials.

\section{REFERENCES}

1. Khokhar HN, Qureshi R. Evaluation of discoloration of some composite restorative materials. Pakistan Oral and Dental Journal 2009;29(1):123-130.

2. Anusavice KJ, Phillip's Science of Dental Materials. 11th ed. Saunders 2004.

3. Khokhar ZA, Razzoog ME. Color stability of restorative resins. Quintessence International 1991;22(9):733-737.

4. Gupta R, Parkash H, Shah N. A spectrophotometric evaluation of color changes of various tooth colored veneering materials after exposure to commonly consumed beverages. The Journal of Indian Prosthodontic Society 2005;5(2):72-78.

5. Thakib A, Al-Shalan. In vitro staining of nanocomposites exposed to a Cola beverage; Pakistan Oral and Dental Journal 2009;29(1):79-84.

6. Topcu FT, Sahinkesen G, Yamanel K. Influence of different drinks on the color stability of dental resin composites. European Journal of Dentistry 2009;350-356.

7. Omata Y, Shigeru UNO, Nakaoki Y. Staining of Hybrid composites with coffee, Oolong tea or red wine. Dental Materials Journal 2006;25(1):125-131.

8. Fontes ST, Fernandez MR. Color stability of nanofilled composite, effect of different immersion media. J Appl Oral Sci 2009;17(5):388-391.

9. Bagheri R, Burrow MF. Influence of food-simulating solutions and surface finish on susceptibility to staining of aesthetic restorative materials. Journal of Dentistry 2005;33:389-398.

10. Mohan M, Shey Z, Vaidyanathan J. Color changes of restorative materials exposed in vitro to cola beverages. Pediatric Dentistry 2008;30(4):309-316.

11. Tahir MM, Woo HTA. Effects of $\mathrm{pH}$ on the microhardness of resin based restorative materials. Operative Dentistry 2005;30(5):661-666. 
12. De Oliveria ALBM. Surface roughness and hardness of a composite resin: influence of finishing and polishing and immersion methods. Material Research 2010; 13(3):409-415.

13. Ergucu Z, Turkun LS. Surface roughness of novel resin composites polished with one-step systems. Operative Dentistry 2007;32(2):185-192.

14. Turkun LS, Turkun M. The effect of one-step polishing system on the surface roughness of three aesthetic resin composite materials. Operative Dentistry 2004;29(2):203-211.

15. Roeder LB, Tate WH, Powers JM. Effect of finishing and polishing procedures on the surface roughness of packable composites. Operative Dentistry 2000;25:534-543.

16. Venturini D, Cenci MS, Demarco FF. Effect of polishing techniques and time on surface roughness, hardness and microleakage of resin composite restorations. Operative Dentistry 2006;31(1):11-17.

17. Schulze KA, Marshal SJ, Marshall GW. Color stability and hardness in dental composites after accelerating aging. Dental materials 2003;19:612-619.

18. Bakar NA, Han L, Akomoto A. Color stability of compomer after immersion in various media. J Esthet Dent 2000;12:258-263.

19. Ahmed KI, Sajjan G. Color stability of ionomer and resin composite restoratives in various environmental solutions: an in vitro Reflection spectrophotometric study. Journal of Conservative Dentistry 2005;8(1):45-51.

20. Ozel E, Korkmaz Y, Attar N. Effect of one-step polishing systems on surface roughness of different flowable restorative materials. Dental Materials Journal 2008;27(6):755-764.

21. Yanikoglu N, Duymus ZY, Yilmaz B. Effects of different solutions on the surface hardness of composite resin materials. Dental Materials Journal 2009;28(3):344-351.

22. Gihan H, Waly 1, El Sharkawy FM. Hydrogen peroxide bleaching: Effects on surface roughness, color and staining susceptibility of microhybrid and nanocomposite. Journal of American Science 2012;8(9):190-199.

23. Cesar Penazzo LEPRI, Regina Guenka PALMA-DIBB. Surface roughness and color change of a composite: influence of beverages brushing. Dental Materials Journal 2012;31(4):689-696.
24. ElMagd DMA, Aziz AAA, Fahmy OI, Taher HAM. In situ Investigation on color change of resin composite restoratives cured by two different curing units. Journal of American Science 2012;8(6):708-715.

25. dos Santos Bertoldo CE, Miranda DA, José Souza E Jr, Aguiar FHB, Lima DANL, Lovadino JR. Evaluation of surface roughness and color stability of direct resin composites after different polishing protocols. International Journal of Dental Clinics 2011:3(3):4-7.

\section{ABOUT THE AUTHORS}

\section{P Sarveshwar Reddy}

Postgraduate Student, Department of Conservative and Endodontics JSS Dental College and Hospital, Mysore, Karnataka, India

\section{KL Sunil Tejaswi (Corresponding Author)}

Reader, Department of Conservative and Endodontics, JSS Dental College and Hospital, Mysore, Karnataka, India, Phone: 8971618243 e-mail: sunilkasipalliendo@rediffmail.com

\section{Suneeth Shetty}

Senior Lecturer, Department of Conservative and Endodontics, JSS Dental College and Hospital, Mysore, Karnataka, India

\section{BM Annapoorna}

Professor, Department of Conservative and Endodontics, JSS Dental College and Hospital, Mysore, Karnataka, India

\section{Sudarshan C Pujari}

Postgraduate Student, Department of Conservative and Endodontics JSS Dental College and Hospital, Mysore, Karnataka, India

\section{HM Thippeswamy}

Reader, Department of Public Health Dentistry, JSS Dental College and Hospital, Mysore, Karnataka, India 